\title{
VERY-HIGH ENERGY PROCESSES IN BLACK HOLE MAGNETOSPHERE: THE CASE OF M87*
}

\author{
STEPHANE VINCENT \\ DESY, Platanenallee 6, 15738 Zeuthen, Germany \\ stephane.vincent@desy.de
}

Received 31 October 2013

Revised 3 December 2013

Published 21 March 2014

\begin{abstract}
M87 is a nearby radio galaxy that is detected at energies ranging from radio to very high energy (VHE) $\gamma$-rays. Its proximity and its jet, misaligned from our line of sight, enable detailed morphological studies. The detection of rapidly variable $\mathrm{TeV}$ emissions on timescale of $\sim 1$ day implies a source of a few Schwarzschild radii $R_{\mathrm{Sch}}$. The $\gamma$-ray telescopes cannot provide images with a sufficient resolution to localize the sites of the $\gamma$-ray production. However, both X-ray and radio observations have shown evidence that charged particles are accelerated in the immediate vicinity of the black hole closer than $100 R_{\mathrm{Sch}}$. We propose that the non-thermal particle acceleration and the VHE emission processes may occur in a pair-starved region of the black hole $(\mathrm{BH})$ magnetosphere. We produce a broadband spectral energy distribution (SED) of the resulting radiation and compare the model with the observed fluxes from the nucleus of M87 for the high $\gamma$-ray activities.
\end{abstract}

Keywords: Galaxies: individual (M87); black hole physics; gamma rays: theory.

PACS Numbers: 95.30.Sf, 95.85.Pw, 98.62.Js

\section{Introduction}

It is generally accepted that the center of the Faranoff-Riley type I (FR-I) radio galaxy M87 harbors a supermassive $\mathrm{BH}$ of mass $\left(6 \pm 0.5 \times 10^{9} \mathrm{M}_{\odot}\right)^{1}$ at a distance of 16.7 $\mathrm{Mpc},{ }^{2}$ which is associated with a kiloparsec scale jet. Due to its size, proximity and orientation, M87 provides a unique opportunity for the study of central active galactic nucleus (AGN) environment. M87 has been observed for a number of years from radio to $\gamma$-rays, and detailed information is available from multi-wavelength collaborations. ${ }^{3}$

M87 shows VHE recurrent activity with variability timescales of a few days or less. The first hint of VHE $\gamma$-ray emission reported by the HEGRA collaboration ${ }^{4}$

* This is an Open Access article published by World Scientific Publishing Company. It is distributed under the terms of the Creative Commons Attribution 3.0 (CC-BY) License. Further distribution of this work is permitted, provided the original work is properly cited. 
triggered extensive observations by the next generation of Imaging Atmospheric Cherenkov Telescopes (IACTs). The H.E.S.S. collaboration firmly established M87 as an emitter above $730 \mathrm{GeV}^{5}$ and revealed flux variability on time-scales of two days, suggesting that the emission region of the $\gamma$-ray is very compact with a dimension similar to the Schwarzschild radius of the central BH. VERITAS also detected VHE $\gamma$-radiation from M87 in $2007^{6}$ above $250 \mathrm{GeV}$ and subsequently monitored the source during the following years (see e.g. Ref. 7). In 2008, H.E.S.S., MAGIC and VERITAS took part in a joint multi-wavelength monitoring campaign of M87 along with Chandra. ${ }^{8}$ The VHE observations were closely coordinated in order to guarantee a reasonable coverage around Chandra pointing and a well-sampled VHE light curve during the first half of 2008. During this joint monitoring campaign, MAGIC reported flaring activities during a 13 day observation period between January 30 and February 11, with day-scale variability occurring throughout the duration of the flare. ${ }^{9}$ Subsequently, the VERITAS collaboration triggered observations of M87, and detected a 4 day flare from February 9 to February 13. The VHE and Chandra $\mathrm{X}$-ray light curves of the joint campaign, along with a coincident VLBA radio light curve, are presented in Ref. 6. The VHE flux monitoring campaign continued in 2009 with MAGIC and VERITAS, and no VHE flaring activity was observed. In April 2010, a bright VHE $\gamma$-ray flare was simultaneously detected by the three ground based Cherenkov telescopes. The detection of the VHE flare triggered further observations in X-ray (Chandra), and radio (43 GHz; VLBA). Chandra observations were taken approximately 2-3 days after the peak of the VHE $\gamma$-ray emission.

The processes by which the high energy radiation is produced, as well as the regions where these processes take place is more debated and has been extensively studied in the past years, based especially on radio, optical, X-ray and $\gamma$-ray observations. The most promising high-energy emission regions are the nucleus and the knot HST-1. At X-ray, Chandra has the angular resolution to separate the two components. ${ }^{11}$ The 2008 and 2010 observations therefore favor the nucleus as the origin of the VHE $\gamma$-radiation. For the 2005 April TeV flaring episode, the data for the nucleus of M87, were severely affected by a "pileup" effect and are not reliable. ${ }^{12}$

After the launch of the Fermi Gamma-ray Space Telescope in the summer 2008, M87 was also detected in the $\mathrm{MeV}-\mathrm{GeV}$ energy range. ${ }^{13}$ The spectral index of $2.26 \pm 0.13$ is consistent with the VHE measurements and no significant variability was found. Following the announcement of a TeV flare from M87 in 2010, the LAT data for the same time period have been checked, and a $95 \%$ confidence upper limit for $E>100 \mathrm{MeV}$ for one day of observation has been calculated (http://fermisky.blogspot.de/2010/02/lat-limit-on-m87-during-tev-flare.html).

\section{Theoretical Interpretations}

The discovery of high-energy $\gamma$-ray emission stimulated an intense theoretical work aimed at clarifying the mechanisms that produce the observed radiation. The rapid $(\sim$ day $)$ variability points to a very compact emission region, ruling out early models 
that invoked emission from large-scale $(\sim \mathrm{kpc})$ jet. ${ }^{14}$ Several theoretical scenarios have been invoked to account for the high energies $(>100 \mathrm{MeV})$ and VHE $\gamma$-ray emission from M87.

In Ref. 15, the authors showed that homogenous one-zone leptonic synchrotron self-Compton (SSC) models are unlikely to explain the observed $\mathrm{TeV}$ spectrum. For an SED whose synchrotron and SSC components peak in the IR and the TeV band, high values of the Doppler factor, $\delta>100$, should be inferred. A solution to this problem is to assume that the jet emission region is structured, as in the decelerating jet model of Ref. 16 or as in the spine-layer model of Ref. 17. Both models are characterized by compact sizes of the VHE $\gamma$-ray emission and no obvious correlation between the low and high-energy emission. They also predict very strong gamma-ray absorption and relatively soft spectrum, and hard spectra are difficult to achieve.

In Ref. 18, the authors present a modification of the one-zone SSC model. In that work, the bulk of the observed emission is proposed to be produced in compact sub-volumes of the main outflow. Such a scenario could in principle account for VHE high states; the individual blob radii of about $10^{14} \mathrm{~cm}$ is compatible with the short variability and the blobs are ejected from the core with reasonable bulk velocities. However, a low magnetic field in the emitting region is required which may be in disagreement with the fact that these regions of the jet are likely strongly magnetized. In Ref. 19, the authors consider mini-jets of energetic particles moving relativistically within the jet. Depending on the direction of motion of the mini-jet, different variability patterns and various variability timescales could, in principle, be expected.

Recently, a different type of modeling has been brought forward to explain the observed VHE $\gamma$-ray emission. In Ref. 20, the authors proposed a scenario where a red giant star interacts with the base of the jet. The VHE emission is due to the interaction of the protons from the jet with the disrupted red giant envelope. To accommodate the light curve and the energy spectrum of the April 2010 flare, a jet power of $\sim 10^{44} \mathrm{erg} . \mathrm{s}^{-1}$ collimated into a narrow jet with semi-opening angle $\theta \sim 2^{\circ}$ are assumed. At the distance from the hole at which the cloud crosses the jet $\sim 10^{16} \mathrm{~cm}$, such a narrow jet would not concur with the VLBI observations showing that the jet opening angle reach $\sim 60^{\circ}$ within 1 milli-arcsecond of the core. ${ }^{21-23}$

The compactness of the particle accelerators operating in the vicinity of the $\mathrm{BH}$ and the absence of a significant cutoff in the spectrum imply that the particle acceleration mechanism is highly efficient. Magnetospheric models, ${ }^{24-27}$ where the non-thermal particle acceleration and emission processes occur at the base of the magnetosphere may then become particularly interesting.

\section{Non-Thermal Processed in BH Magnetosphere}

The most natural location for emitting regions with a size of the order of a few Schwarzschild radii is the immediate vicinity of the $\mathrm{BH}$. Theoretical studies of $\mathrm{BH}$ 
magnetospheres have shown that the electromagnetic field can be used to extract the rotational energy of BHs. First, Wald ${ }^{28}$ found the solution for the electromagnetic field in the vicinity of a Kerr $\mathrm{BH}$ immersed in an uniform magnetic field aligned with the hole's rotation axis in free space. As a consequence of the coupling of the magnetic field to frame dragging effects, an electric field arises. In the absence of currents flow to slow down the $\mathrm{BH}$ and to extract its rotational energy, no outflow of energy at infinity is possible. Therefore, it is necessary to have a magnetosphere filled with charges and currents to extract the rotational energy of the hole. Then Blandford \& Znajek ${ }^{29}$ developed the general theory of force-free magnetosphere filled with plasma and found a steady-state solution. As they pointed out, charged particles can reach a high enough energy to radiate VHE photons by synchrotron radiation and inverse Compton scattering. These photons can encounter thermal photons and create electron-positron pairs which will in turn be accelerated leading to a cascade. Such a mechanism of $\gamma$-ray production from the vicinity of a $\mathrm{BH}$ is analogous to the mechanism of $\gamma$-ray emission from a rotation-powered pulsar. Recent 3D-relativistic MHD simulations of BH accretion disks and magnetospheres have shown that the Blandford-Znajek solution is stable and the rotational energy of BHs can indeed be extracted electromagnetically (see e.g. Refs. 30-33).

\subsection{Particle acceleration and $\mathrm{VHE}$ emission in the BH magnetosphere}

A BH embedded in an external magnetic field $B$ and rotating with an angular velocity $\Omega_{\mathrm{H}}=a c / 2 R_{\mathrm{H}}$ will generate an electric potential difference across a gap height $h$, given by ${ }^{34}$

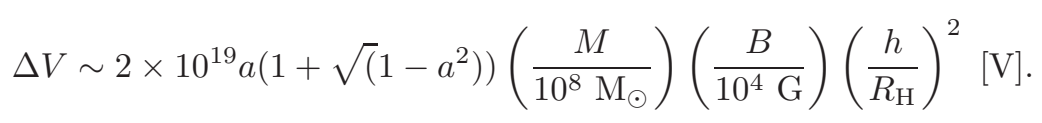

Charged particles that get injected into the gap will accelerate and quickly reach a terminal Lorentz factor at which radiative losses balance energy gain, i.e., $e \Delta V=$ $\left(P_{\text {Sync }}+P_{\mathrm{IC}}\right) h / c$, where $P_{\text {Sync }}$ is the synchrotron losses

$$
P_{\text {Sync }}=\frac{4}{3} \sigma_{\mathrm{T}} c \gamma^{2} U_{\mathrm{B}}
$$

and $P_{\mathrm{IC}}$ the inverse Compton losses

$$
P_{\mathrm{IC}}=\frac{4}{3} \sigma_{\mathrm{T}} c \gamma^{2} U_{\mathrm{rad}}
$$

If the ambient photon field is weak enough, as expected in M87, the energy loss of an electron will be dominated by IC radiation, limiting achievable Lorentz factor to $\gamma_{\mathrm{IC}} \sim 4 \times 10^{8}$. The maximum energy of IC photons up-scattered by the accelerated electrons can exceed $\gamma_{\mathrm{IC}} m_{\mathrm{e}} c^{2} \simeq 200 \mathrm{TeV}$. At such energies, interactions with the ambient radiation field can initiate pair cascades just above the gap. 


\subsection{Numerical modeling and application to the flaring Te $V$ emission}

The location of the gap in the BH magnetosphere depends on the structure of both the accretion flow and the magnetic field near the event horizon. Despite intensive theoretical study of the physics of $\mathrm{BH}$ magnetospheres, the details of the geometry remain uncertain.

We have developed a numerical code that allows, for a given geometry of the gap and configuration of the magnetic field, a quantitative study of the energy distributions of electrons accelerated in the gap and associated electromagnetic radiation. We assume that the gap occupies a spherical layer above the $\mathrm{BH}$. The geometry of the electromagnetic field is assumed to be given by the split-monopole solution. The electrons are assumed to be initially at rest with respect to the locally inertial observer and injected at the base of the gap. Trajectories are numerically integrated taking into account the effects of general relativity and energy losses due to synchrotron and IC scattering. The spectrum of IC radiation is calculated by tracing the photon trajectories through the Kerr spacetime metric from the emission point to infinity. In the case of M87, we show that the 2008 and 2010 observed TeV $\gamma$-ray emission can be explained by electron acceleration and $\gamma$-ray production in a compact region close to the event horizon. In Fig. 1, we show both the spectral energy distribution (SED) of the synchrotron and the inverse Compton scattered radiation. We report the emission from the magnetosphere model relative to the high states observed in 2008 (grey lines) and 2010 (black lines) for our model parameters $B=0.03 \mathrm{G}$, luminosity of the background radiation $L_{\mathrm{IR}}=10^{41} \mathrm{erg} . \mathrm{s}^{-1}$, energy of

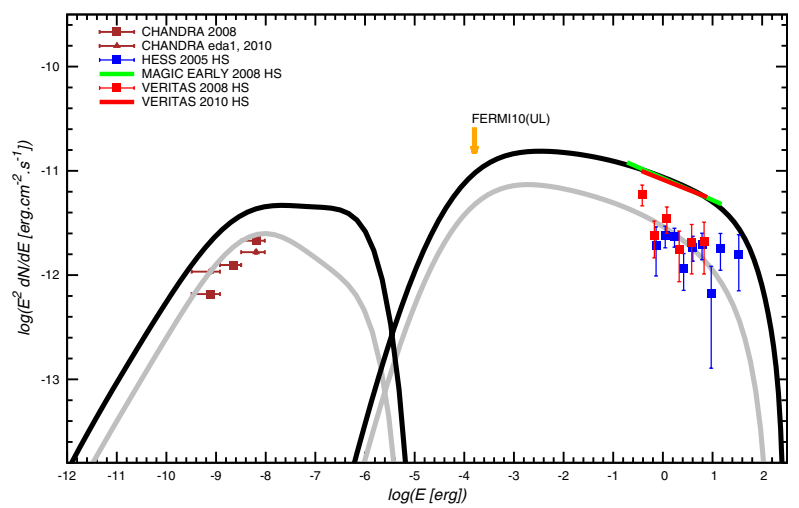

Fig. 1. Model outputs for the spectral energy distribution. The observed emission includes: (1) Chandra observation in 2008, labelled with the solid filled brown squares with error bars, and Chandra observation on 2010 April 1 (solid filled brown circles with error bars); (2) Fermi-LAT upper limit during the 2010 flare indicated by an orange arrow (http:/ fermisky.blogspot.de/2010/02/latlimit-on-m87-during-tev-flare.html); (3) HESS observations in 2005, which are shown with the filled blue squares with error bars; (4) MAGIC best-fit spectrum for data taken on 2008 February 1 in green curve; (5) VERITAS spectrum during the 2008 February flare represented by red filled squares and the fit function reported during the 2010 April flare shown in red line. 
the background photons for the inverse Compton scattering $E_{\mathrm{IR}}=4 \times 10^{-14} \mathrm{erg}$, size of the background radiation $R_{\mathrm{IR}}=20 R_{\mathrm{Sch}}$, and the rotation-induced electric field $E=500-2 \times 10^{3} \mathrm{~V} \cdot \mathrm{m}^{-1}$. Because M87 accretes from magnetized hot phase, density and magnetic field will further increase at smaller radii. The lack of information about the accretion regime leads to a significant uncertainty in the plasma density near the event horizon. Assuming that the accreting matter moves at relativistic speeds and that the magnetic field is generated by the accreting matter, one can find that maximum possible magnetic field is about $15 \mathrm{G}$. Hence if the magnetic field scales as $\propto r^{-2}$ as for a split-monopole field geometry, it implies that the gap is located at a distance of $20 R_{\mathrm{Sch}}$ above the $\mathrm{BH}$.

\section{Acknowledgments}

I acknowledge support through the Helmholtz Alliance for Astroparticle Particle.

\section{References}

1. Gebhardt, K., \& Thomas, J. 2009, ApJ, 700, 1690.

2. Mei, S., Blakeslee, J. P., Côté, P., et al., 2007, ApJ, 655, 144.

3. Abramowski, A., Acero, F., Aharonian, F., et al., 2012, ApJ, 746, 151.

4. Aharonian, F., Akhperjanian, A., Beilicke, M., et al., 2003, A\&GA, 403, L1.

5. Aharonian, F., Akhperjanian, A. G., Bazer-Bachi, A. R., et al., 2006, Science, 314, 1424.

6. Acciari, V. A., Beilicke, M., Blaylock, G., et al., 2008, ApJ, 679, 397.

7. Acciari, V. A., Aliu, E., Arlen, T., et al., 2010, ApJ, 716, 819.

8. Beilicke, M., Hui, C. M., Mazin, D., et al., 2008, American Institute of Physics Conference Series, 1085, 553.

9. Albert, J., Aliu, E., Anderhub, H., et al., 2008, ApJL, 685, L23.

10. Acciari, V. A., Aliu, E., Arlen, T., et al., 2009, Science, 325, 444.

11. Harris, D. E., Cheung, C. C., Stawarz, Ł., Biretta, J. A., \& Perlman, E. S. 2009, ApJ, 699, 305.

12. Harris, D. E., Massaro, F., Cheung, C. C., et al., 2011, ApJ, 743, 177.

13. Abdo, A. A., Ackermann, M., Ajello, M., et al., 2009, ApJ, 707, 55.

14. Stawarz, Ł., Sikora, M., \& Ostrowski, M. 2003, ApJ, 597, 186.

15. Georganopoulos, M., Perlman, E. S., \& Kazanas, D. 2005, ApJL, 634, L33.

16. Georganopoulos, M., \& Kazanas, D. 2003, ApJL, 589, L5.

17. Tavecchio, F., \& Ghisellini, G. 2008, MNRAS, 385, L98.

18. Lenain, J.-P., Boisson, C., Sol, H., \& Katarzyński, K. 2008, A\&̇A, 478, 111.

19. Giannios, D., Uzdensky, D. A., \& Begelman, M. C. 2010, MNRAS, 402, 1649.

20. Barkov, M. V., Aharonian, F. A., \& Bosch-Ramon, V. 2010, ApJ, 724, 1517.

21. Junor, W., Biretta, J. A., \& Livio, M. 1999, Nature, 401, 891.

22. Ly, C., Walker, R. C., \& Junor, W. 2007, ApJ, 660, 200.

23. Asada, K., \& Nakamura, M. 2012, ApJL, 745, L28.

24. Neronov, A., \& Aharonian, F. A. 2007, ApJ, 671, 85.

25. Rieger, F. M., \& Aharonian, F. A. 2008, International Journal of Modern Physics D, 17,1569 .

26. Vincent, S., \& LeBohec, S. 2010, MNRAS, 409, 1183.

27. Levinson, A., \& Rieger, F. 2011, ApJ, 730, 12. 
28. Wald, R. M. 1974, PRD, 10, 1680.

29. Blandford, R. D., \& Znajek, R. L. 1977, MNRAS, 179, 433.

30. Komissarov, S. S. 2001, MNRAS, 326, L41.

31. Komissarov, S. S. 2004, MNRAS, 350, 1431.

32. McKinney, J. C. 2006, MNRAS, 367, 1797.

33. Penna, R. F., Narayan, R., \& Sạdowski, A. 2013, MNRAS, 2531.

34. Thorne, K. S., Price, R. H., \& MacDonald, D. A. 1986, Black Holes: The Membrane Paradigm. 